\title{
Normal Forms for Submanifolds under Group Actions
}

\author{
Peter J. Olver ${ }^{\dagger}$ \\ School of Mathematics \\ University of Minnesota \\ Minneapolis, MN 55455 \\ olver@umn.edu \\ http: //www.math.umn.edu/ olver
}

\begin{abstract}
We describe computational algorithms for constructing the explicit power series expansions for normal forms of submanifolds under transformation groups. The procedure used to derive the coefficients relies on the recurrence formulae for differential invariants provided by the method of equivariant moving frames.
\end{abstract}

\section{Introduction.}

The equivariant method of moving frames, introduced in [4], provides a powerful computational tool for investigating the equivalence and symmetry properties of submanifolds under general Lie group actions (and, more generally, infinite-dimensional Lie pseudo-groups, $[\mathbf{2 3}, \mathbf{2 4}]$ ), and determining the required differential invariants. The main new tool is the recurrence relations, which completely prescribe the structure of the noncommutative differential algebra they generate through the process of invariant differentiation. Remarkably, these relations and the consequent differential algebraic structure can be completely and straightforwardly constructed, requiring only basic linear algebra,

$\dagger$ Supported in part by NSF Grant DMS 11-08894.

June 23, 2018 
and can thus be readily implemented in any modern computer algebra system, including Mathematica, Maple, and Sage.

A simple example is provided by the Euclidean geometry of space curves $C \subset \mathbb{R}^{3}$, under the action of the group of rigid motions - translations and rotations. The fundamental differential invariants are the curvature and torsion of the space curve, and the invariant differential operator is differentiation with respect to arc length. As a consequence, every Euclidean differential invariant can be expressed as a function of curvature, torsion, and their successive arc-length derivatives.

The normalization procedure underlying the construction of a moving frame is equivalent to the specification of a "normal form" for submanifolds under the group action. Roughly, to construct a normal form, one uses the group transformations to simplify, as much as possible, the Taylor expansion of the submanifold at a given point. The result will be called a normal form for the submanifold at the point, also known as the Monge or Monge-Taylor form, $[\mathbf{1}, \mathbf{3}]$. As we note below, this simplification is exactly the same as the choice of cross-section to the prolonged group orbits, which is the first step in the equivariant moving frame construction. Once a normal form has been specified, the nonconstant coefficients in the resulting Taylor series expansion form a complete system of differential invariants, known, in the equivariant approach, as the fundamental normalized differential invariants.

The purpose of the present note is to explain, in simplified form, the moving frame algorithms and recurrence formulae, and how they can be used to construct the normal form expansion of a submanifold in terms of the fundamental differential invariants and their invariant derivatives. While direct calculations can be very tedious, if not impossible due to the limitations of current computer algebra software and hardware, the recurrence formulae provide a simple, straightforward route to the desired formulae. In this paper, we describe this calculus, first in the simplest context of plane curves, and then for general submanifolds under Lie group actions. The results are illustrated by a few basic examples of geometric and imaging importance.

\section{Plane Curves.}

For simplicity, we first describe the normal form construction in the its most basic manifestation: plane curves under "ordinary" group actions. The general version can be found below in Section 3 .

Throughout this section, $C \subset M=\mathbb{R}^{2}$ will denote a regular, $\operatorname{smooth}^{\dagger}\left(\mathrm{C}^{\infty}\right)$ plane curve. We use $z=(x, u)$ as local coordinates on $M$, and $t \in I \subset \mathbb{R}$ as a curve parameter, so that $C$ is the image of the function $z(t)=(x(t), u(t))$ for $t$ in the interval $I$. Regularity requires that the curve's tangent vector is nowhere vanishing ${ }^{\ddagger}: d z / d t=\left(x_{t}, u_{t}\right) \neq \mathbf{0}$. We will identify parametrizations that have identical image curves, meaning that we allow reparametrization, including those that reverse orientation. In particular, the curve is a

$\dagger$ One can apply the construction to curves of class $\mathrm{C}^{n}$ provided $n$ is sufficiently large that all derivatives indicated are continuous.

$\ddagger$ Subscripts on dependent variables indicate derivatives. 
graph if it is parametrized by the horizontal coordinate $x$, so that $z(x)=(x, u(x))$ for $x \in I \subset \mathbb{R}$. Locally, in a neighborhood of $z_{0}=\left(x_{0}, u_{0}\right) \in C$, a curve can be parametrized uniquely as a graph if and only if it intersects the vertical fiber $\left\{x=x_{0}\right\}$ transversally, meaning that its tangent vector at $z_{0}$ is not vertical, i.e., $x_{t} \neq 0$ there.

Given a graph defined by the function $u(x)$, we will identify its Taylor polynomial of order $n$ at a point $z_{0}=\left(x_{0}, u_{0}\right)=\left(x_{0}, u\left(x_{0}\right)\right) \in C$, namely ${ }^{\dagger}$,

$$
u\left(x_{0}\right)+u_{x}\left(x_{0}\right)\left(x-x_{0}\right)+\frac{1}{2} u_{x x}\left(x_{0}\right)\left(x-x_{0}\right)^{2}+\cdots+\frac{1}{n !} u_{n}\left(x_{0}\right)\left(x-x_{0}\right)^{n},
$$

with the $n^{\text {th }}$ order jet of the curve at the point $z_{0}$. Note that the $n$ jet is uniquely prescribed by the derivatives of order $\leq n$ at the point in question. Thus, the space of $n^{\text {th }}$ order transverse $\mathrm{f}^{\ddagger}$ curve jets, denoted $\mathrm{J}^{n}$, can be identified with $\mathbb{R}^{n+2}$, with coordinates

$$
z^{(n)}=\left(x, u, u_{x}, u_{x x}, \ldots, u_{n}\right) .
$$

The $n$-jet of the graph $C=\{(x, u(x))\}$ at the point $z_{0}=\left(x_{0}, u\left(x_{0}\right)\right) \in C$ is thereby identified with the $(n+2)$-tuple

$$
\left.\mathrm{j}_{n} C\right|_{z_{0}}=\left(x_{0}, u\left(x_{0}\right), u_{x}\left(x_{0}\right), u_{x x}\left(x_{0}\right), \ldots, u_{n}\left(x_{0}\right)\right) \in \mathrm{J}^{n} .
$$

One can straightforwardly derive, via implicit differentiation, expressions for the curve jet components (2.2) in terms of a general parametrization $z(t)=(x(t), u(t))$, writing the $n^{\text {th }}$ order jet coordinate $u_{n}$ as an explicit rational function of the derivatives, of order $\leq n$, of $x(t), u(t)$. For example,

$$
u_{x}=D_{x} u=\frac{u_{t}}{x_{t}}, \quad u_{x x}=D_{x} u_{x}=\frac{1}{x_{t}} D_{t}\left(\frac{u_{t}}{x_{t}}\right)=\frac{x_{t} u_{t t}-u_{t} x_{t t}}{x_{t}^{3}}, \quad \cdots,
$$

with the higher order expressions obtained by iteratively applying the implicit total derivative operator

$$
D_{x}=\frac{1}{x_{t}} D_{t} .
$$

By a differential function, we mean a (locally defined) real-valued function on the jet space, $F: \mathrm{J}^{n} \rightarrow \mathbb{R}$, and so, in coordinates, taking form

$$
F\left(z^{(n)}\right)=F\left(x, u, u_{x}, u_{x x}, \ldots, u_{n}\right) .
$$

To us, the most important differential functions are the differential invariants, e.g., curvature, torsion, and the like. Note that one can use the parametric differentiation formulae (2.3) to re-express any differential function in terms of a general curve parametrization.

Let $G$ be an $r$-dimensional Lie group acting on $M=\mathbb{R}^{2}$. There is an induced action of $G$ on curves, with $g \in G$ mapping the curve $C$ parametrized by $z(t)$ to the image curve $\widetilde{C}=g \cdot C$ parametrized by $\tilde{z}(t)=g \cdot z(t)$. Two curves $C, \widetilde{C} \subset M$ are said to be equivalent if

$\dagger$ In this section, $u_{n}$ represents the $n^{\text {th }}$ order derivative of $u$ with respect to $x$.

$\ddagger$ See $[\mathbf{1 7}]$ for the extended jet bundle construction, that includes non-transverse curves. 
there exists a group element $g \in G$ such that $\widetilde{C}=g \cdot C$. Again, we allow reparametrization in our identification of curves. In practice, we are primarily interested in local equivalence, in the neighborhood of corresponding points on the two curves.

The action of $G$ on curves induces an action on their jets. In other words, given a jet $\left.z_{0}^{(n)} \in \mathrm{J}^{n}\right|_{z_{0}}$, let $C$ be any transverse curve whose jet at $z_{0} \in C$ coincides with $z_{0}^{(n)}$ at the point $z_{0} \in C$. Then $g \cdot z_{0}^{(n)}$ is equal to the $n$-jet of the image curve $\widetilde{C}=g \cdot C$ at the image point $\tilde{z}_{0}=g \cdot z_{0}$. If the image curve is not transverse, the action is not defined in the ordinary jet space (although it is defined on the extended jet bundle, cf. [17]), meaning that the prolonged group action on $\mathrm{J}^{n}$ is, in general, only a local action even if the action on $M$ is global. The explicit formulae for the prolonged action of a transformation group are obtained by implicit differentiation, $[\mathbf{4}, \mathbf{1 7}]$.

A differential invariant of order $n$ is a differential function $I\left(z^{(n)}\right)$ that is unaffected by the prolonged group action, i.e., $I\left(g \cdot z^{(n)}\right)=I\left(z^{(n)}\right)$ for all $g \in G$ and all $z^{(n)} \in \mathrm{J}^{n}$, where defined. Clearly, equivalent curves have identical differential invariants, although, of course, their explicit formulae in terms of the curves' individual parametrizations may vary. The Cartan solution to the equivalence problem, $[\mathbf{1 8}]$, is based on the functional identities, or syzygies, among the differential invariants which are used to parametrize the associated signature. (In the case of curves in Euclidean space, the signature curve was introduced earlier by Bruce and Giblin, [1] , under the name "Monge-Taylor map".) See, for example, $[\mathbf{2}, \mathbf{7}, \mathbf{8}, \mathbf{1 0}, \mathbf{1 6}, \mathbf{2 5}]$ for various applications of the differential invariant signature to object recognition in digital images.

In its simplest incarnation, a cross-section to the prolonged group action is a fixed jet $z_{0}^{(n)} \in \mathrm{J}^{n}$ with the property that for any (nearby) curve $C$ and point $z \in C$ there is a unique group element $g \in G$ such that

$$
g \cdot\left(\left.\mathrm{j}_{n} C\right|_{z}\right)=\left.\mathrm{j}_{n}(g \cdot C)\right|_{z_{0}}=z_{0}^{(n)},
$$

meaning that the group element maps the curve jet at $z$ to the fixed cross-section jet. In particular $g \cdot z=z_{0}$. A straightforward chain rule argument demonstrates that the group element satisfying (2.5) depends only of the $n$-jet $z^{(n)}=\left.\mathrm{j}_{n} C\right|_{z}$ of the curve at the point $z$. In view of uniqueness, we write $g=\rho\left(z^{(n)}\right)$, whereby (2.5) is equivalent to the equation

$$
\rho\left(z^{(n)}\right) \cdot z^{(n)}=z_{0}^{(n)} .
$$

In the language of [4], the map ${ }^{\dagger} \rho: \mathrm{J}^{n} \rightarrow G$ defines a (right) moving frame of order $n$, and, as can be easily proved, satisfies the right equivariance rule

$$
\rho\left(g \cdot z^{(n)}\right)=\rho\left(z^{(n)}\right) \cdot g^{-1},
$$

where the dot on the left hand side indicates the prolonged group action on $\mathrm{J}^{n}$, while the dot on the right hand side represents group multiplication. Occasionally, formulae are

$\dagger$ Typically $\rho$ is only defined on an open subset of the jet space. 
more simply written in terms of the corresponding left equivariant moving frame, which is merely the group inverse of the right moving frame:

$$
\widetilde{\rho}\left(z^{(n)}\right)=\rho\left(z^{(n)}\right)^{-1}, \quad \text { satisfying the left equivariance rule } \tilde{\rho}\left(g \cdot z^{(n)}\right)=g \cdot \widetilde{\rho}\left(z^{(n)}\right) \text {, }
$$

and mapping the normal form jet to the curve jet: $\widetilde{\rho}\left(z^{(n)}\right) \cdot z_{0}^{(n)}=z^{(n)}$.

Given a choice of cross-section, a curve $C_{0}$ is said to be in normal form if $z_{0} \in C_{0}$ and its $n$-jet at $z_{0}$ coincides with the fixed cross-section jet: $\left.\mathrm{j}_{n} C_{0}\right|_{z_{0}}=z_{0}^{(n)}$. Thus, given

$$
z_{0}^{(n)}=\left(x_{0}, u_{0}=c_{0}, c_{1}, \ldots, c_{n}\right)
$$

any normal form curve, parametrized as the graph of the function $u_{0}(x)$, has Taylor expansion

$$
\begin{aligned}
u_{0}(x)=c_{0} & +c_{1}\left(x-x_{0}\right)+\frac{1}{2} c_{2}\left(x-x_{0}\right)^{2}+\cdots+\frac{1}{n !} c_{n}\left(x-x_{0}\right)^{n} \\
& +\frac{1}{(n+1) !} u_{n+1}\left(x_{0}\right)\left(x-x_{0}\right)^{n+1}+\frac{1}{(n+2) !} u_{n+2}\left(x_{0}\right)\left(x-x_{0}\right)^{n+2}+\cdots,
\end{aligned}
$$

at $x=x_{0}$, whose first $n+1$ coefficients are fixed by the choice of cross-section jet, whereas the values of those of order $\geq n+1$ depend upon the particularities of the curve $C_{0}$.

Remark: Existence of a cross-section of the above type is equivalent to the transitivity and freeness ${ }^{\dagger}$ of the prolonged group action on an open subset of $\mathrm{J}^{n}$. If the Lie group $G$ has dimension $r$, then this requires $n=r-2$. A planar group action that admits a crosssection in the above sense is known as ordinary, [18]. The only non-ordinary group actions on $\mathbb{R}^{2}$ are intransitive actions and those whose prolongations exhibit pseudo-stabilization, meaning that they act intransitively but not freely on some jet space. All "standard" transitive group actions arising in geometry and image processing are ordinary. Moreover, non-ordinary actions can be readily handled by the general moving frame construction described in the following section.

Applying the moving frame group element $g=\rho\left(z^{(n)}\right)$ to the curve $C$ produces the normal form curve $C_{0}=g \cdot C=\rho\left(z^{(n)}\right) \cdot C$ associated with the point $z \in C$, that satisfies the normal form constraint $\mathrm{j}_{n} C_{0}=z_{0}^{(n)}$. Clearly, two curves are locally equivalent if and only if they have identical normal forms at the matching points. Consequently, each Taylor coefficient of the normal form curve at the point $z_{0}$, when expressed as a function of the original curve jet, defines a differential invariant. In other words, for any $k$,

$$
z_{0}^{(k)}=\left.\mathrm{j}_{k} C_{0}\right|_{z_{0}}=\left.\mathrm{j}_{k}\left(\rho\left(z^{(n)}\right) \cdot C\right)\right|_{z_{0}}=\rho\left(z^{(n)}\right) \cdot\left(\left.\mathrm{j}_{k} C\right|_{z}\right)=\rho\left(z^{(n)}\right) \cdot z^{(k)}=I^{(k)}\left(z^{(k)}\right),
$$

$\dagger$ The action of $G$ is free at $z^{(n)} \in \mathrm{J}^{n}$ if the only group element that fixes $z^{(n)}$ is the identity, i.e., $g \cdot z^{(n)}=z^{(n)}$ if and only if $g=e$. 
defines a vector-valued differential invariant: $I^{(k)}\left(g \cdot z^{(k)}\right)=I^{(k)}\left(z^{(k)}\right)$ for all $g \in G$ where defined, whose individual components provide $k+2$ scalar-valued differential invariants ${ }^{\ddagger}$ :

$$
\begin{aligned}
I^{(k)}\left(z^{(k)}\right) & =\left(H\left(z^{(k)}\right), I_{0}\left(z^{(k)}\right), I_{1}\left(z^{(k)}\right), \ldots, I_{k}\left(z^{(k)}\right)\right) \\
& =\left(x_{0}, c_{0}, c_{1}, \ldots, c_{n}, I_{n+1}\left(z^{(n+1)}\right), \ldots, I_{k}\left(z^{(k)}\right)\right) .
\end{aligned}
$$

As a result, the normal form Taylor expansion (2.9) is

$$
\begin{aligned}
u_{0}(x)=c_{0} & +c_{1}\left(x-x_{0}\right)+\frac{1}{2} c_{2}\left(x-x_{0}\right)^{2}+\cdots+\frac{1}{n !} c_{n}\left(x-x_{0}\right)^{n}+ \\
& +\frac{1}{(n+1) !} I_{n+1}\left(z^{(n+1)}\right)\left(x-x_{0}\right)^{n+1}+\cdots+\frac{1}{k !} I_{k}\left(z^{(k)}\right)\left(x-x_{0}\right)^{k}+\cdots,
\end{aligned}
$$

We will call $I_{j}\left(z^{(j)}\right)$ the $j^{\text {th }}$ order normalized differential invariant; note that its value is independent of the choice of $k \geq j$ in (2.11); indeed, it would be convenient to set $k=\infty$ and work with Taylor series (infinite jets) throughout. Of course, the first $n+2$ of these, $H, I_{0}, \ldots, I_{n}$, are constant, and are known as the phantom invariants, since they equal the corresponding Taylor coefficient (2.11) of the cross-section jet: $I_{j}\left(z^{(n)}\right)=c_{j}$.

According to $[\mathbf{4}]$, the non-phantom or fundamental normalized differential invariants of order $>n$, namely $I_{n+1}\left(z^{(n+1)}\right), I_{n+2}\left(z^{(n+2)}\right), \ldots$, form a complete system of differential invariants for the action of $G$ on curves, meaning that, locally, any other differential invariant can be written, uniquely, as a function thereof. Indeed, the Replacement Rule states that if $J\left(z^{(k)}\right)=J\left(x, u, u_{x}, \ldots, u_{k}\right)$ is any differential invariant of $\operatorname{order}^{\dagger} k>n$, then, replacing each of its arguments by the corresponding normalized invariant,

$$
J\left(z^{(k)}\right)=J\left(x_{0}, c_{0}, \ldots, c_{n}, I_{n+1}\left(z^{(n+1)}\right), \ldots, I_{k}\left(z^{(k)}\right)\right)
$$

gives an explicit formula for $J$ in terms of the fundamental normalized invariants. In symbolic computation terminology, [11], (2.13) is a rewrite rule expressing any differential invariant in terms of the fundamental generators.

Further, the moving frame map induces a process of invariantization, denote by $\iota$, that associates a differential invariant with any differential function. Namely, if $F\left(z^{(k)}\right)$ is any function of the curve jets, then its invariantization $J\left(z^{(k)}\right)=\iota\left[F\left(z^{(k)}\right)\right]$ is the unique differential invariant that agrees with the value of $F$ on the normal form prescribed by the cross-section: $J\left(z_{0}^{(k)}\right)=F\left(z_{0}^{(k)}\right)$. Note that invariantization respects all algebraic operations - but not differentiation, which is the point of the recurrence formulae derived below. It is not hard to see that the invariantization process is readily implemented by substituting each jet coordinate appearing in the argument of $F$ by the corresponding normalized differential invariant:

$$
\iota\left[F\left(z^{(k)}\right)\right]=F\left(x_{0}, c_{0}, \ldots, c_{n}, I_{n+1}\left(z^{(n+1)}\right), \ldots, I_{k}\left(z^{(k)}\right)\right) .
$$

$\ddagger$ When $k \leq n$, then $I^{(k)}=\left(x_{0}, c_{0}, c_{1}, \ldots, c_{k}\right)$ is constant.

$\dagger$ Since $G$ acts transitively on $\mathrm{J}^{n}$, any differential invariant of order $\leq n$ is necessarily constant, and still satisfies the Replacement Rule. 
Furthermore, invariantization does not affect differential invariants: $\iota\left[J\left(z^{(k)}\right)\right]=J\left(z^{(k)}\right)$ and hence, comparison with (2.14) immediately establishes the Replacement Rule (2.13).

Example 2.1. Plane curves under orientation-preserving rigid motions: In this example, $G=\mathrm{SE}(2)$ is the special Euclidean group, consisting of translations and rotations of $\mathbb{R}^{2}$ :

$$
x \mapsto x \cos \phi-u \sin \phi+a, \quad u \mapsto x \cos \phi+u \sin \phi+b, \quad a, b \in \mathbb{R}, \quad-\pi<\phi \leq \pi .
$$

To place a plane curve in Euclidean normal form at a point $z \in C$, we first use the translations to move the base point to the origin, $x_{0}=u_{0}=0$, and then rotate the translated curve so that its tangent is horizontal, whereby $u_{x, 0}=0$. The resulting Euclidean normal form for a plane curve has Taylor expansion

$$
u_{0}(x)=\frac{1}{2} I_{2} x^{2}+\frac{1}{6} I_{3} x^{3}+\frac{1}{24} I_{4} x^{4}+\cdots+\frac{1}{k !} I_{k} x^{k}+\cdots
$$

at the origin. Its Taylor coefficients

$$
I_{k}=\iota\left(u_{k}\right), \quad k \geq 2,
$$

when expressed in terms of the original curve parametrization, are the fundamental normalized differential invariants.

The preceding choice of normal form corresponds to the cross-section

$$
x=u=u_{x}=0,
$$

whence the three associated phantom invariants are

$$
\iota(x)=H=0, \quad \iota(u)=I_{0}=0, \quad \iota\left(u_{x}\right)=I_{1}=0 .
$$

The resulting left moving frame $\mathrm{e}^{\dagger} \widetilde{\rho}: \mathrm{J}^{1} \rightarrow \mathrm{SE}(2)$ can be identified with the classical moving frame, $[\mathbf{9}]$, namely, its translation component is the point $z \in C$, while the columns of the rotation matrix, $R=[\mathbf{t}, \mathbf{n}]$, are the orthonormal frame vectors based at $z$, that is, the unit tangent $\mathbf{t}$ and normal $\mathbf{n}$. Furthermore, by direct computation or, alternatively, by applying the moving frame construction, the lowest order normalized differential invariant

$$
I_{2}=\iota\left(u_{x x}\right)=\kappa=\frac{u_{x x}}{\left(1+u_{x}^{2}\right)^{3 / 2}}
$$

turns out to be the Euclidean curvature of the curve. We defer the identification of the higher order normalized invariants $I_{3}, I_{4}, \ldots$, until we have constructed the associated recurrence formulae.

$\dagger$ Typically, while the right moving frame (2.7) plays a more fundamental role and is easier to compute, in classical geometries, the corresponding left moving frame (2.8) includes the usual frame vectors on the submanifold, cf. [4]. 
Remark: In the preceding example, there remains an unresolved discrete ambiguity since we can rotate by $\pi$ radians without affecting the cross-section (2.18). The effect is to map $u_{0}(x)$ to $-u_{0}(-x)$, and hence change the sign of the even order normalized invariants, $I_{2 j} \mapsto-I_{2 j}$, so that, in particular, the curvature invariant changes sign: $\kappa \mapsto-\kappa$. This can be avoided by either working with its absolute value or, if one restricts attention to closed curves, by fixing the orientation. Here, to avoid technicalities, we will ignore this final ambiguity (as is done in most treatments), referring to [20] for the full details, including the additional effects of reflections on the moving frame and differential invariants.

An alternative method of generating differential invariants is through invariant differentiation. Given a transformation group acting on plane curves, we use $d s$ to denote the $G$-invariant arc-length element, or, equivalently, the invariant ${ }^{\dagger}$ one-form of lowest order. We remark that the invariant one-forms can also be systematically constructed through a reasonably straightforward extension of the invariantization process associated with the moving frame, and refer the reader to $[\mathbf{4 , 2 2}]$ for details.

Let $\mathcal{D}=d / d s$ be the dual invariant differential operator, i.e., the arc length derivative. Invariance of the arc-length form $d s$ implies that $\mathcal{D}$ maps a differential invariant of order $k$ to a differential invariant of order $k+1$. In particular, starting with the (non-constant) normalized differential invariant $\kappa=I_{n+1}$ of lowest order, namely $n+1$, which we identify ${ }^{\ddagger}$ as the $G$-invariant curvature function, its successive arc-length derivatives $\kappa_{s}=\mathcal{D} \kappa, \kappa_{s s}=$ $\mathcal{D}^{2} \kappa, \ldots$, are differential invariants of respective orders $n+2, n+3, \ldots$ It is known that they also generate the algebra of differential invariants; one way of proving this assertion is by inspection of the recurrence formulae. The Replacement Rule (2.13) tells us that these are all functions of the normalized differential invariants; vice versa, it can be shown that the normalized differential invariants are themselves certain functions of the curvature invariant and its successive arc length derivatives. The resulting formulae

$$
I_{k}=F_{k}\left(\kappa, \kappa_{s}, \ldots, \kappa_{k-n-1}\right), \quad k \geq n+1,
$$

enable one to express the coefficients of the normal form Taylor expansion (2.12) of a curve in terms of the curvature invariant and its arc-length derivatives. Our goal is to develop the machinery that enables one to straightforwardly compute these formulas, and hence the explicit Taylor expansion for the normal form of a curve under a group action.

While, in principle, knowing the explicit coordinate formulae for the curvature invariants enables one, e.g. via the Replacement Rule (2.13), to express them in terms of the normalized invariants, and hence, by inversion, determine the desired formulae (2.21), in

$\dagger$ Strictly speaking, $d s$ is only "contact-invariant", meaning that it is not an invariant form on jet space but, rather, is invariant when restricted to curve jets, or, equivalently, is invariant modulo contact forms, [18].

$\ddagger$ Identification of $\kappa$ with a classical geometric quantity (Euclidean curvature, equi-affine curvature, projective curvature, etc.) requires an appropriate choice of normal form. Other choices may result in some function, e.g., a constant multiple, of the classical curvature invariant. Incidentally, the function in question can be straightforwardly found by applying the Replacement Rule (2.13) to the classical formula. 
practice, for complicated group actions and higher order invariants, this can be a very cumbersome and complicated procedure that can overwhelm the abilities of even sophisticated computer algebra systems such as Mathematica, Maple, Sage, etc. The power of the equivariant moving frame method is that it enables one to systematically and straightforwardly derive these formulae without a priori knowledge of the explicit formulae for any of the differential invariants, or the invariant arc length derivative, or even the moving frame itself. All that is required is the formulae for the prolonged infinitesimal generators of the group action, coupled with some simple (symbolic) linear algebra!

To explain the computational algorithm, let

$$
\mathbf{v}_{\sigma}=\xi_{\sigma}(x, u) \frac{\partial}{\partial x}+\varphi_{\sigma}(x, u) \frac{\partial}{\partial u}, \quad \sigma=1, \ldots, r
$$

be a basis for the Lie algebra of infinitesimal generators of the action of $G$, which are vector fields on $M,[\mathbf{1 7}]$. Let

$$
\operatorname{pr} \mathbf{v}_{\sigma}=\xi_{\sigma}(x, u) \frac{\partial}{\partial x}+\sum_{k \geq 0} \varphi_{\sigma}^{k}\left(x, u^{(k)}\right) \frac{\partial}{\partial u_{k}}, \quad \sigma=1, \ldots, r,
$$

be the corresponding infinitesimal generators of the prolonged action of $G$ on the jet spaces, whose coefficients are explicitly determined by the well-known prolongation formula, $[\mathbf{1 7}]$ :

$$
\varphi_{\sigma}^{k}\left(z^{(k)}\right)=D_{x}^{k}\left[\varphi_{\sigma}(x, u)-\xi_{\sigma}(x, u) u_{x}\right]+\xi_{\sigma}(x, u) u_{k+1} .
$$

Here

$$
D_{x}=\frac{\partial}{\partial x}+u_{x} \frac{\partial}{\partial u}+u_{x x} \frac{\partial}{\partial u_{x}}+u_{x x x} \frac{\partial}{\partial u_{x x}}+\cdots
$$

is the total derivative operator, which effectively differentiates differential functions by treating $u$ as a function of $x$.

The recurrence formulae for the differentiated invariants, [4], are

$$
I_{k+1}=\mathcal{D} I_{k}-\sum_{\sigma=1}^{r} K_{\sigma} \iota\left[\varphi_{\sigma}^{k}\left(x, u^{(k)}\right)\right], \quad k=0,1,2, \ldots,
$$

where $\iota$ is the invariantization map (2.14) and $K_{1}, \ldots, K_{r}$ are certain as yet unspecified differential invariants known as the Maurer-Cartan invariants ${ }^{\dagger}$. In particular, if one takes $0 \leq k \leq n=r-2$ in (2.26), then $I_{k}=c_{k}$ is a constant phantom invariant, and hence the first term on the right hand side of the recurrence formula is zero. Thus, the result is a system of $r-1$ linear equations for the $r$ Maurer-Cartan invariants in terms of the normalized differential invariants of order $\leq r-1=n+1$. These are supplemented by the

$\dagger$ This is because they are, in fact, the coefficients of the pull-backs of the Maurer-Cartan forms via the moving frame map $\rho: \mathrm{J}^{n} \rightarrow G$, [4]. However, while this is essential to proving the validity of (2.26), from a purely practical standpoint there is no need to know this theoretical fact, or even understand what a "Maurer-Cartan form" is, since, as we will soon see, we can readily determine their explicit formulae directly from the recurrence formulae themselves. 
recurrence formula for the remaining phantom invariant $H=\iota(x)=x_{0}$, which takes the form

$$
1=\mathcal{D} H-\sum_{\sigma=1}^{r} K_{\sigma} \iota\left[\xi_{\sigma}(x, u)\right]=-\sum_{\sigma=1}^{r} K_{\sigma} \iota\left[\xi_{\sigma}(x, u)\right] .
$$

It can be shown that the resulting system of $r$ linear equations can be uniquely solved for the Maurer-Cartan invariants $K_{1}, \ldots, K_{r}$, which can thus all be expressed as certain rational functions of the curvature invariant $\kappa=I_{n+1}$. With these expressions in hand, the resulting higher order recurrence formulae $(2.26)$, for $k>n$, will then iteratively provide the required formulae (2.21) for each $I_{k+1}$ in terms of the arc length derivatives of $\kappa$. Let us see how this works in the context of a couple of examples.

Example 2.2. Return to the action of the Euclidean group on plane curves introduced in Example 2.1. We use

$$
\mathcal{D}=D_{s}=\frac{1}{\sqrt{1+u_{x}^{2}}} D_{x}
$$

to denote the invariant arc length total derivative operator.

The infinitesimal generators of the action (2.15) are

$$
\mathbf{v}_{1}=\partial_{x}, \quad \mathbf{v}_{2}=\partial_{u}, \quad \mathbf{v}_{3}=-u \partial_{x}+x \partial_{u} .
$$

Applying the prolongation formula (2.24), the infinitesimal generators of the prolonged action of $\mathrm{SE}(2)$ on plane curves are

$$
\begin{aligned}
\operatorname{pr} \mathbf{v}_{1}=\partial_{x}, \quad \operatorname{pr} \mathbf{v}_{2} & =\partial_{u} \\
\operatorname{pr} \mathbf{v}_{3}=-u \partial_{x}+x \partial_{u}+(1 & \left.+u_{x}^{2}\right) \partial_{u_{x}}+3 u_{x} u_{x x} \partial_{u_{x x}}+\left(4 u_{x} u_{x x x}+3 u_{x x}^{2}\right) \partial_{u_{x x x}}+ \\
& +\left(5 u_{x} u_{x x x x}+10 u_{x x} u_{x x x}\right) \partial_{u_{x x x x}}+ \\
& +\left(6 u_{x} u_{x x x x x}+15 u_{x x} u_{x x x x}+10 u_{x x x}^{2}\right) \partial_{u_{x x x x x}}+\cdots
\end{aligned}
$$

Thus, the recurrence formulae $(2.26,27)$ for the three phantom invariants $(2.19)$ are

$$
\begin{aligned}
1 & =\mathcal{D} H-K_{1} \iota(1)-K_{2} \iota(0)-K_{3} \iota(-u)=-K_{1}, \\
0=I_{1} & =\mathcal{D} I_{0}-K_{1} \iota(0)-K_{2} \iota(1)-K_{3} \iota(x)=-K_{2}, \\
\kappa=I_{2} & =\mathcal{D} I_{1}-K_{1} \iota(0)-K_{2} \iota(0)-K_{3} \iota\left(1+u_{x}^{2}\right)=-K_{3},
\end{aligned}
$$

and hence the Maurer-Cartan invariants are

$$
K_{1}=-1, \quad K_{2}=0, \quad K_{3}=-\kappa .
$$

Using $(2.17,19)$, these values are then substituted into the higher order recurrence formulae (2.26) to produce

$$
\begin{aligned}
& I_{3}=\mathcal{D} I_{2}-K_{3} \iota\left(3 u_{x} u_{x x}\right)=\kappa_{s}, \\
& I_{4}=\mathcal{D} I_{3}-K_{3} \iota\left(4 u_{x} u_{x x x}+3 u_{x x}^{2}\right)=\kappa_{s s}+3 \kappa^{3}, \\
& I_{5}=\mathcal{D} I_{4}-K_{3} \iota\left(5 u_{x} u_{x x x x}+10 u_{x x} u_{x x x}\right)=\kappa_{s s s}+19 \kappa^{2} \kappa_{s}, \\
& I_{6}=\mathcal{D} I_{5}-K_{3} \iota\left(6 u_{x} u_{x x x x x}+15 u_{x x} u_{x x x x}+10 u_{x x x}^{2}\right)=\kappa_{s s s s}+34 \kappa^{2} \kappa_{s s}+48 \kappa \kappa_{s}^{2}+45 \kappa^{5},
\end{aligned}
$$


and so on. We conclude that the explicit Taylor expansion of a curve placed in Euclidean normal form (2.16) is

$$
\begin{aligned}
u_{0}(x)=\frac{1}{2} \kappa x^{2}+\frac{1}{6} \kappa_{s} x^{3}+\frac{1}{24}( & \left.\kappa_{s s}+3 \kappa^{3}\right) x^{4}+\frac{1}{5 !}\left(\kappa_{s s s s}+19 \kappa^{2} \kappa_{s}\right) x^{5}+ \\
& +\frac{1}{6 !}\left(\kappa_{\text {ssss }}+34 \kappa^{2} \kappa_{s s}+48 \kappa \kappa_{s}^{2}+45 \kappa^{5}\right) x^{6}+\cdots .
\end{aligned}
$$

Higher order terms can be systematically constructed by continuing the above procedure. However, I do not know a general formula for the differential polynomials in $\kappa$ that appear as coefficients.

Example 2.3. A more substantial example is provided by the geometry of equiaffine planar curves, $[\mathbf{9}]$, also of importance for image processing, [2]. The equi-affine group $\mathrm{SA}(2)$ acts on $M=\mathbb{R}^{2}$ via area-preserving affine transformations

$$
g \cdot(x, u)=(\alpha x+\beta u+a, \gamma x+\delta u+b), \quad \alpha \delta-\beta \gamma=1 .
$$

The normalization equations

$$
x=u=u_{x}=0, \quad u_{x x}=1, \quad u_{x x x}=0,
$$

define a cross-section to the prolonged action, which leads to the classical equi-affine moving frame. This normalization can be applied except at inflection points, i.e., provided the nondegeneracy condition $u_{x x} \neq 0$ holds. (Similar nondegeneracy conditions appear in most examples, the preceding case of Euclidean plane curves being a notable exception. At isolated inflection points one can, in principle, use the general moving frame procedure, to be presented in Section 3, to construct a higher order moving frame.) The cross-section (2.35) corresponds to the following equi-affine normal form for a non-degenerate plane curve:

$$
u_{0}(x)=\frac{1}{2} x^{2}+\frac{1}{4 !} I_{4} x^{4}+\frac{1}{5 !} I_{5} x^{5}+\frac{1}{6 !} I_{6} x^{6}+\cdots .
$$

The fundamental differential invariant is the equi-affine curvature

$$
\kappa=I_{4}=\iota\left(u_{x x x x}\right)=\frac{u_{x x} u_{x x x x}-\frac{5}{3} u_{x x x}^{2}}{u_{x x}^{8 / 3}},
$$

while

$$
\mathcal{D}=\iota\left(D_{x}\right)=u_{x x}^{-1 / 3} D_{x}
$$

is the invariant differentiation operator with respect to equi-affine arc-length. Both formulas $(2.37,38)$ can be straightforwardly found by a complete implementation of the moving frame construction, but are not required to perform the ensuing computations.

Our goal is to write the higher order differential invariants

$$
I_{k}=\iota\left(u_{k}\right), \quad k \geq 4,
$$

and hence the equi-affine normal form (2.36), in terms of the equi-affine curvature and its arc-length derivatives. Applying $(2.23,24)$, the prolonged infinitesimal generators for the 
equi-affine group action (2.34) are

$$
\begin{aligned}
\mathbf{v}_{1} & =\partial_{x}, \\
\mathbf{v}_{2} & =\partial_{u}, \\
\mathbf{v}_{3} & =-x \partial_{x}+u \partial_{u}+2 u_{x} \partial_{u_{x}}+3 u_{x x} \partial_{u_{x x}}+4 u_{x x x} \partial_{u_{x x x}}+5 u_{x x x x} \partial_{u_{x x x x}}+\cdots, \\
\mathbf{v}_{4} & =u \partial_{x}-u_{x}^{2} \partial_{u_{x}}-3 u_{x} u_{x x} \partial_{u_{x x}}-\left(4 u_{x} u_{x x x}+3 u_{x x}^{2}\right) \partial_{u_{x x x}}- \\
& -\left(5 u_{x} u_{x x x x}+10 u_{x x} u_{x x x}\right) \partial_{u_{x x x x}}+\cdots \\
\mathbf{v}_{5} & =x \partial_{u}+\partial_{u_{x}} .
\end{aligned}
$$

Thus, the recurrence formulae $(2.26,27)$ for the phantom invariants coming from invariantizing the cross-section coordinates (2.37) are

$$
\begin{gathered}
1=\mathcal{D} H-K_{1}=-K_{1}, \quad 0=I_{1}=\mathcal{D} I_{0}-K_{2}=-K_{2}, \quad 1=I_{2}=\mathcal{D} I_{1}-K_{5}=-K_{5}, \\
0=I_{3}=\mathcal{D} I_{2}-3 K_{3}=-3 K_{3}, \quad \kappa=I_{4}=\mathcal{D} I_{3}+3 K_{4}=3 K_{4},
\end{gathered}
$$

and hence the Maurer-Cartan invariants are

$$
K_{1}=-1, \quad K_{2}=0, \quad K_{3}=0, \quad K_{4}=\frac{1}{3} I_{4}=\frac{1}{3} \kappa, \quad K_{5}=-1 .
$$

These values are then substituted into the higher order recurrence formulae (2.26) to iteratively produce the desired formulae:

$$
\begin{aligned}
& I_{5}=\mathcal{D} I_{4}=\kappa_{s}, \\
& I_{6}=\mathcal{D} I_{5}+5 I_{4}^{2}=\kappa_{s s}+5 \kappa^{2}, \\
& I_{7}=\mathcal{D} I_{6}+7 I_{4} I_{5}=\kappa_{s s s}+17 \kappa \kappa_{s}, \\
& I_{8}=\mathcal{D} I_{7}+\frac{28}{3} I_{4} I_{6}+\frac{35}{3} I_{4}^{3}=\kappa_{s s s s}+\frac{79}{3} \kappa \kappa_{s s}+17 \kappa_{s}^{2}+\frac{175}{3} \kappa^{3},
\end{aligned}
$$

and so on. We conclude that the equi-affine normal form for a plane curve at a noninflection point is given by

$$
\begin{aligned}
u_{0}(x)=\frac{1}{2} x^{2}+\frac{1}{4 !} \kappa x^{4}+\frac{1}{5 !} \kappa_{s} x^{5} & +\frac{1}{6 !}\left(\kappa_{s s}+5 \kappa^{2}\right) x^{6}+\frac{1}{7 !}\left(\kappa_{s s s}+17 \kappa \kappa_{s}\right) x^{7}+ \\
& +\frac{1}{8 !}\left(\kappa_{\text {ssss }}+\frac{79}{3} \kappa \kappa_{s s}+17 \kappa_{s}^{2}+\frac{175}{3} \kappa^{3}\right) x^{8}+\cdots .
\end{aligned}
$$

Again, while they are easily found by iterating the preceding algorithm, I do not know a general explicit formula for the differential polynomials appearing in the normal form expansion (2.43).

\section{Normal Forms for Submanifolds.}

We now turn to the equivariant moving frame construction, [4], that applies to completely general Lie group actions and, when suitably adapted, $[\mathbf{2 3}]$, also to infinitedimensional Lie pseudo-group actions. Let $M$ be an $m$-dimensional manifold which, since we are working locally, we identify as (an open subset of) $\mathbb{R}^{m}$. Given $1 \leq p<m$, there is an induced action of $G$ on $p$-dimensional submanifolds $S \subset M$, and we are interested in determining when two such submanifolds are equivalent, meaning that there exists $g \in G$ mapping one (locally) to the other: $\widetilde{S}=g \cdot S$. As before, we are interested in the submanifold purely as a subset of $M$, and thus allow arbitrary reparametrizations thereof. 
(Although one can readily adapt the procedure to avoid or restrict allowable reparametrizations.) The solution to the equivalence problem is based on the differential invariant signature, and the moving frame method allows one to explicitly determine the fundamental differential invariants used to construct the required signature, $[\mathbf{2}, \mathbf{4}]$.

We employ coordinates $(x, u)=\left(x^{1}, \ldots, x^{p}, u^{1}, \ldots, u^{q}\right)$ on $\mathbb{R}^{m}$, with $p+q=m$, treating the $x$ 's as independent variables and the $u$ 's as dependent variables, whereby any $p$-dimensional submanifold $S$ that is transverse to the vertical fibers $\left\{x=x_{0}\right\}$ can be locally identified with the graph, $S=\{(x, u(x))\}$, of a smooth vector-valued function $x \mapsto u(x)$ with components $u^{\alpha}\left(x^{1}, \ldots, x^{p}\right), \alpha=1, \ldots, q$. We identify the $n^{\text {th }}$ order Taylor expansion of $u(x)$ at a point $x_{0}$ in its domain as the $n$ jet of the submanifold at the base point $z_{0}=\left(x_{0}, u_{0}\right)=\left(x_{0}, u\left(x_{0}\right)\right) \in S$. The resulting $n^{\text {th }}$ order jet space $\mathrm{J}^{n}$, for $p$-dimensional submanifolds, is coordinatized by the independent variables $x^{1}, \ldots, x^{p}$, the dependent variables $u^{1}, \ldots, u^{q}$, and their derivatives up to order $n$, which we denote by $u_{J}^{\alpha}$, with $\alpha=1, \ldots, q$, and $J=\left(j_{1}, \ldots, j_{k}\right)$ a symmetric multi-index, with $1 \leq j_{k} \leq p$, of order $1 \leq k=\# J \leq n$, whose entries indicate partial derivatives of $u^{\alpha}$ with respect to the $x$ 's. Thus, a point in $\mathrm{J}^{n}$ is specified by the coordinates

$$
z^{(n)}=\left(\ldots x^{i} \ldots u^{\alpha} \ldots u_{J}^{\alpha} \ldots\right), \quad \text { where } i=1, \ldots, p, \quad \alpha=1, \ldots, q, \quad \# J \leq n .
$$

See $[4, \mathbf{1 7}, \mathbf{1 8}]$ for details.

The action of $G$ on submanifolds induces an action on their jets, leading to the prolonged group action on jet space. Explicit formulas are obtained by implicit differentiation. In general, a cross-section is a submanifold of the jet space, $\mathcal{K} \subset \mathrm{J}^{n}$ that has complementary dimension and is transverse to the prolonged group orbits. Moreover, we assume that, for each jet $z^{(n)} \in \mathrm{J}^{n}$ sufficiently close to $\mathcal{K}$, there is a unique group element $g=\rho\left(z^{(n)}\right)$ that maps $z^{(n)}$ to the cross-section, which, as before, specifies the moving frame map ${ }^{\dagger}$ $\rho: \mathrm{J}^{n} \rightarrow G$, satisfying the right equivariance condition (2.7). Transversality means that no (non-zero) prolonged infinitesimal generator is tangent to the cross-section, which can be straightforwardly verified using their explicit formulas, cf. (3.7) below, and involves computing the rank of a certain matrix. Existence of a cross-section, and hence a moving frame, requires that the prolonged action be (locally) free and regular on an open subset of $\mathrm{J}^{n}$, and it can be proved that, assuming the action on $M$ is locally effective on subsets, local freeness holds at a sufficiently high order $n,[\mathbf{1 9}]$. Usually - although not always, $[\mathbf{1 3}, \mathbf{1 5}]$ — one chooses a coordinate cross-section obtained by setting (or normalizing) $r=\operatorname{dim} G$ of the jet coordinates equal to suitable constants. Almost always, one chooses a minimal order cross-section, meaning that the normalized jet coordinates have as low an order as possible. For example, the cross-section for an ordinary planar group action used in Section 2 is minimal. From here on we implicitly assume that we have chosen a coordinate cross-section of minimal order, although the general moving frame constructions can be readily adapted to more exotic choices.

If the group acts transitively on $M$, a minimal order coordinate cross-section is contained in the jet space over a single point $\left.\mathcal{K} \subset \mathrm{J}^{n}\right|_{z_{0}}$. One can interpret such a coordinate cross-section as normalizing particular Taylor coefficients of the submanifolds passing

$\dagger$ As before, the notation allows $\rho$ to be only defined on an open subset of $\mathrm{J}^{n}$. 
through the base point $z_{0}$ - which is almost always taken to be at the origin. Once the moving frame map is specified, the normal form for a submanifold $S \subset M$ at a point $z \in S$ is obtained by applying the moving frame map corresponding to the submanifold's $n$-jet at the point in question, $z^{(n)}=\left.\mathrm{j}_{n} S\right|_{z}$, so that $S_{0}=\rho\left(z^{(n)}\right) \cdot S$ is a submanifold passing through $z_{0} \in S_{0}$ and whose jet belongs to the cross-section, i.e., whose Taylor coefficients corresponding to the normalized cross-section jet coordinates have been normalized to the specified values. The remaining jet coordinates (Taylor coefficients), when expressed in terms of the originating submanifold jets $z^{(k)}$, provide a complete system of differential invariants, known as the normalized differential invariants

With the moving frame in hand, we define the invariantization of a differential function $F\left(z^{(n)}\right)$ to be the unique differential invariant $J\left(z^{(n)}\right)=\iota\left[F\left(z^{(n)}\right)\right]$ that agrees with $F$ on the cross-section: $F|\mathcal{K}=J| \mathcal{K}$. In particular, invariantization of the jet coordinate functions leads to the normalized differential invariants:

$$
H^{i}=\iota\left(x^{i}\right), \quad I_{J}^{\alpha}=\iota\left(u_{J}^{\alpha}\right) .
$$

The $r$ jet coordinates that are used to define the cross-section produce the constant, phantom differential invariants, and the remaining, non-phantom fundamental normalized invariants provide a complete system of functionally independent differential invariants. The invariantization map has the explicit formula

$$
\iota\left[F\left(\ldots x^{i} \ldots u_{J}^{\alpha} \ldots\right)\right]=F\left(\ldots H^{i} \ldots I_{J}^{\alpha} \ldots\right),
$$

in which one replaces all jet coordinates by the corresponding normalized differential invariants. Moreover, invariantization clearly preserves differential invariants, $\iota(J)=J$, and hence any differential invariant can be expressed in terms of the normalized differential invariants via the Replacement Rule:

$$
J\left(\ldots x^{i} \ldots u_{J}^{\alpha} \ldots\right)=J\left(\ldots H^{i} \ldots I_{J}^{\alpha} \ldots\right) .
$$

Furthermore, for $p$-dimensional submanifolds there are $p$ invariant differential operators $\mathcal{D}_{1}, \ldots, \mathcal{D}_{p}$ that map differential invariants to differential invariants, and obtained by invariantizing ${ }^{\dagger}$ the corresponding total derivative operators

$$
D_{i}=\frac{\partial}{\partial x^{i}}+\sum_{\alpha=1}^{q} \sum_{J} u_{J, i}^{\alpha} \frac{\partial}{\partial u_{J}^{\alpha}}, \quad i=1, \ldots, p,
$$

where $u_{J, i}^{\alpha}=D_{i}\left(u_{J}^{\alpha}\right)=u_{j_{1} \ldots j_{k} i}^{\alpha}$. The Basis Theorem, $[\mathbf{4}, \mathbf{2 4}]$, states that there exist a finite number of generating differential invariants $J_{1}, \ldots, J_{l}$ with the property that any other differential invariant can be written as a (not necessarily uniquely specified) function of the generating invariants and their successive invariant derivatives,

$$
J_{k, I}=\mathcal{D}_{i_{1}} \cdots \mathcal{D}_{i_{n}} J_{k}, \quad k=1, \ldots, l, \quad 1 \leq i_{\nu} \leq p, \quad n \geq 0 .
$$

$\dagger$ More correctly, one invariantizes the basic horizontal one-forms, $\varpi^{i}=\iota\left(d x^{i}\right)$, producing a invariant horizontal coframe, and the invariant differential operators are the dual total differentiation operators. 
In particular, one can express all the normalized differential invariants in terms of them, and the explicit formulae can be found by iteratively applying the recurrence formulae, to be described next. It is known that, given a moving frame $\rho: \mathrm{J}^{n} \rightarrow G$ of order $n$, the nonconstant normalized differential invariants of order $\leq n+1$ form a generating set, although it typically contains redundancies and one can, by inspection of the recurrence relations and the commutation formulae (see Example 3.2 below) among the invariant differential operators, produce a smaller generating set. Determining the minimal number $l=l_{\text {min }}$ of generating differential invariants is a very challenging problem, with surprises even in seemingly well-studied situations, $[\mathbf{2 1}, \mathbf{1 2}]$. The case of curves is, however, known, where the answer (for ordinary group actions) is precisely $l=m-1$; see [6] for intriguing Lie theoretic tools for determining their orders.

To write out the recurrence formulae, let

$$
\mathbf{v}_{\sigma}=\sum_{i=1}^{p} \xi_{\sigma}^{i}(x, u) \frac{\partial}{\partial x^{i}}+\sum_{\alpha=1}^{q} \varphi_{\sigma}^{\alpha}(x, u) \frac{\partial}{\partial u^{\alpha}}, \quad \sigma=1, \ldots, r,
$$

be a basis for the infinitesimal generators of the action of $G$ on $M$. The corresponding prolonged infinitesimal generators for the action on the jet spaces are given by the wellknown prolongation formula

$$
\operatorname{pr} \mathbf{v}_{\sigma}=\sum_{i=1}^{p} \xi_{\sigma}^{i}(x, u) \frac{\partial}{\partial x^{i}}+\sum_{\alpha=1}^{q} \sum_{\# J \geq 0} \varphi_{J, \sigma}^{\alpha}\left(x, u^{(n)}\right) \frac{\partial}{\partial u_{J}^{\alpha}},
$$

whose coefficients are readily calculated:

$$
\varphi_{J}^{\alpha}=D_{J}\left[\varphi^{\alpha}-\sum_{i=1}^{p} \xi^{i} u_{i}^{\alpha}\right]+\sum_{i=1}^{p} \xi^{i} u_{J, i}^{\alpha}
$$

where $D_{J}=D_{j_{1}} \cdot \ldots \cdot D_{j_{k}}$, with $J=\left(j_{1}, \ldots, j_{k}\right), 1 \leq j_{\nu} \leq p$, denotes the corresponding higher order total derivative.

The general recurrence formula for differential invariants ${ }^{\dagger}$ can be then formulated as follows. Let $F\left(z^{(n)}\right)$ be any differential function. Then

$$
\iota\left(D_{i} F\right)=\mathcal{D}_{i} \iota(F)-\sum_{\sigma=1}^{r} K_{i}^{\sigma} \iota\left[\operatorname{pr} \mathbf{v}_{\sigma}(F)\right], \quad i=1, \ldots, p,
$$

where $^{\ddagger} K_{i}^{\sigma}$ are certain differential invariants known as the Maurer-Cartan invariants. (Our earlier equations $(2.26,27)$ are both special cases of $(3.9)$, in which $F=u_{k}$ and $x$, respectively.) In particular, if we take $F$ to be one of the cross-section coordinates, then its invariantization is a constant phantom invariant, and hence the first term on the hand side of (3.9) is zero. Thus, fixing $1 \leq i \leq p$, and then successively substituting the $r$

$\dagger$ This is a special case of the more general recurrence formula for differential forms, $[\mathbf{4}, \mathbf{1 4}]$.

$\ddagger$ Now we have made the group index $\sigma$ on the Maurer-Cartan invariants a superscript. 
cross-section coordinates into (3.9) produces a system of $r=\operatorname{dim} G$ linear equations which, according to [4], can be uniquely solved for the Maurer-Cartan invariants $K_{i}^{1}, \ldots, K_{i}^{r}$ as rational functions of the normalized differential invariants. Substituting these expressions, for all $i=1, \ldots, p$, into (3.9), where now $F$ is taken to be successive non-normalized jet coordinates, produces the full system of recurrence relations that completely specifies the structure of the rational, non-commutative differential invariant algebra and, in particular, leads to the desired formulae for the Taylor coefficients as invariant derivatives of the generating differential invariants.

Let us illustrate the procedure with two further examples.

Example 3.1. Consider the $r=6$ - dimensional Euclidean group SE(3) acting by rigid motions on space curves $C \subset M=\mathbb{R}^{3}$. Here the submanifolds have dimension $p=1$, and we use coordinates $z=(x, u, v)$ on $M$. As usual, we concentrate on curves given by the graphs of functions: $u=u(x), v=v(x)$, although all our results can be readily adapted to general parametrized curves $z(t)=(x(t), u(t), v(t))^{T}$. Indeed, the recurrence formulae and consequent relations among differential invariants make no reference as to how the curve is parametrized. On the other hand, when writing out explicit formulas for the differential invariants, we use

$$
z_{t}=\left(\begin{array}{c}
x_{t} \\
u_{t} \\
v_{t}
\end{array}\right)=\left(\begin{array}{c}
1 \\
u_{x} \\
v_{x}
\end{array}\right), \quad z_{t t}=\left(\begin{array}{c}
x_{t t} \\
u_{t t} \\
v_{t t}
\end{array}\right)=\left(\begin{array}{c}
0 \\
u_{x x} \\
v_{x x}
\end{array}\right), \quad z_{t t t}=\left(\begin{array}{c}
x_{t t t} \\
u_{t t t} \\
v_{t t t}
\end{array}\right)=\left(\begin{array}{c}
0 \\
u_{x x x} \\
v_{x x x}
\end{array}\right),
$$

and so on, to denote the derivative vectors along the curve, where the second expression can be used in the special case of a graph, parametrized by $t=x$.

A basis for the infinitesimal generators is provided by the vector fields

$$
\begin{array}{lll}
\mathbf{v}_{1}=\partial_{x}, & \mathbf{v}_{2}=\partial_{u}, & \mathbf{v}_{3}=\partial_{v}, \\
\mathbf{v}_{4}=v \partial_{u}-u \partial_{v}, & \mathbf{v}_{5}=-u \partial_{x}+x \partial_{u}, & \mathbf{v}_{6}=-v \partial_{x}+x \partial_{v} .
\end{array}
$$

Applying the prolongation formula $(3.7,24)$ leads to the corresponding prolonged infinitesimal generators on the curve jet spaces, parametrized by $x, u, v, u_{x}, v_{x}, u_{x x}, v_{x x}, u_{x x x}, \ldots$. To order 3 , we find

$$
\begin{aligned}
\operatorname{pr} \mathbf{v}_{1}= & \partial_{x}, \\
\operatorname{pr} \mathbf{v}_{2}= & \partial_{u}, \\
\operatorname{pr} \mathbf{v}_{3}= & \partial_{v}, \\
\operatorname{pr} \mathbf{v}_{4}= & v \partial_{u}-u \partial_{v}+v_{x} \partial_{u_{x}}-u_{x} \partial_{v_{x}}+v_{x x} \partial_{u_{x x}}-u_{x x} \partial_{v_{x x}}+v_{x x x} \partial_{u_{x x x}}-u_{x x x} \partial_{v_{x x x}}+\cdots, \\
\operatorname{pr} \mathbf{v}_{5}=-u \partial_{x}+x \partial_{u}+\left(1+u_{x}^{2}\right) \partial_{u_{x}}+u_{x} v_{x} \partial_{v_{x}}+3 u_{x} u_{x x} \partial_{u_{x x}}+\left(u_{x x} v_{x}+2 u_{x} v_{x x}\right) \partial_{v_{x x}}+ & \\
& \quad+\left(4 u_{x} u_{x x x}+3 u_{x x}^{2}\right) \partial_{u_{x x x}}+\left(u_{x x x} v_{x}+3 u_{x x} v_{x x}+3 u_{x} v_{x x x}\right) \partial_{v_{x x x}}+\cdots, \\
\operatorname{pr} \mathbf{v}_{6}=-v \partial_{x}+x \partial_{v}+u_{x} v_{x} \partial_{u_{x}}+\left(1+v_{x}^{2}\right) \partial_{v_{x}}+\left(2 u_{x x} v_{x}+u_{x} v_{x x}\right) \partial_{u_{x x}}+3 v_{x} v_{x x} \partial_{v_{x x}}+ & \\
& \quad+\left(3 u_{x x x} v_{x}+3 u_{x x} v_{x x}+u_{x} v_{x x x}\right) \partial_{u_{x x x}}+\left(4 v_{x} v_{x x x}+3 v_{x x}^{2}\right) \partial_{v_{x x x}}+\cdots .
\end{aligned}
$$

The classical moving frame, $[\mathbf{9}]$, relies on the normalization equations

$$
x=0, \quad u=0, \quad v=0, \quad u_{x}=0, \quad v_{x}=0, \quad v_{x x}=0,
$$


which serve to define a coordinate cross-section provided $u_{x x} \neq 0$. (Indeed, the classical moving frame is not defined at inflection points of the space curve.) This corresponds to translating and rotating the curve into the Euclidean normal form so that it goes through the origin, has tangent in the direction of the $x$-axis, and second order contact with the $x, u$ plane. For this particular cross-section, the translational component of the left moving frame is the point on the curve, $z=(x, u, v) \in C$, while the columns of the rotational component $R=[\mathbf{t}, \mathbf{n}, \mathbf{b}] \in \mathrm{SO}(3)$ are the usual orthonormal tangent, normal, and binormal frame vectors based at $z$. However, keep in mind that these explicit identifications are not required to generate the recurrence formulae for the differential invariants.

We let

$$
H=\iota(x), \quad I_{k}=\iota\left(u_{k}\right), \quad J_{k}=\iota\left(v_{k}\right),
$$

be the normalized differential invariants resulting from invariantization, so that, in view of (3.13), the phantom invariants are

$$
\begin{array}{lll}
H=\iota(x)=0, & I_{0}=\iota(u)=0, & J_{0}=\iota(v)=0, \\
I_{1}=\iota\left(u_{x}\right)=0, & J_{1}=\iota\left(v_{x}\right)=0, & J_{2}=\iota\left(v_{x x}\right)=0 .
\end{array}
$$

One can further identify

$$
I_{2}=\iota\left(u_{x x}\right)=\kappa, \quad J_{3}=\iota\left(v_{x x x}\right)=\kappa \tau
$$

with, respectively, the classical curvature invariant ${ }^{\dagger}$, and the product of curvature and torsion. These two invariants generate the differential invariant algebra through invariant differentiation with respect to arc length, and the recurrence formulae allow one to express the normalized invariants $I_{k}, J_{k}$ in terms of curvature, torsion, and their successive arclength derivatives: $\kappa, \tau, \kappa_{s}, \tau_{s}, \ldots$ We note the classical formulas

$$
\begin{aligned}
\kappa=\frac{\left\|z_{t} \times z_{t t}\right\|}{\left\|z_{t}\right\|^{3}} & =\frac{\sqrt{\left(u_{x} v_{x x}-u_{x x} v_{x}\right)^{2}+u_{x x}^{2}+v_{x x}^{2}}}{\left(1+u_{x}^{2}+v_{x}^{2}\right)^{3 / 2}}, \\
\tau=\frac{z_{t} \times z_{t t} \cdot z_{t t t}}{\left\|z_{t} \times z_{t t}\right\|^{2}} & =\frac{u_{x x} v_{x x x}-u_{x x x} v_{x x}}{\left(u_{x} v_{x x}-u_{x x} v_{x}\right)^{2}+u_{x x}^{2}+v_{x x}^{2}}, \\
d s=\left\|z_{t}\right\| d t \quad & =\sqrt{1+u_{x}^{2}+v_{x}^{2}} d x,
\end{aligned}
$$

which can be obtained by fully implementing the moving frame construction, [5]. The first expression is valid for arbitrary parametrized curves, and the second is for graphs. However, we emphasize that these explicit formulas are not required for us to determine the recurrence formulas, and hence the Taylor coefficients of the Euclidean normal form of a space curve.

In this example, the recurrence formulae (3.9) have the form

$$
\iota\left(D_{x} F\right)=\mathcal{D} \iota(F)-\sum_{\sigma=1}^{6} K_{\sigma} \iota\left(\operatorname{pr} \mathbf{v}_{\sigma}(F)\right)
$$

$\dagger$ As in the planar version, there is an ambiguous sign resulting from a $180^{\circ}$ rotation, and one usually sets $\kappa=\left|I_{2}\right|$ to ensure full invariance. To avoid minor technicalities, we shall ignore this extra complication here, and refer the reader to $[\mathbf{2 0}]$ for further details. 
for any differential function $F\left(x, u, v, u_{x}, v_{x}, u_{x x}, \ldots\right)$, where $K_{1}, \ldots, K_{6}$ are the MaurerCartan invariants. Taking $F$ in (3.18) to be, in turn, each of the cross-section jet coordinates $x, u, v, u_{x}, v_{x}, v_{x x}$ that define the phantom invariants (3.15) leads, via (3.12), to the linear system

$$
\begin{aligned}
1 & =\mathcal{D} H-K_{1}=-K_{1}, & 0=I_{1} & =\mathcal{D} I_{0}-K_{2}=-K_{2}, \\
I_{2} & =\mathcal{D} I_{1}-K_{5}=-K_{5}, & 0=J_{1} & =\mathcal{D} J_{0}-K_{3}=-K_{3}, \\
0=J_{2} & =\mathcal{D} J_{1}-K_{6}=-K_{6}, & J_{3} & =\mathcal{D} J_{2}-I_{2} K_{4}=-I_{2} K_{4},
\end{aligned}
$$

which can be immediately solved for the Maurer-Cartan invariants:

$$
K_{1}=-1, \quad K_{2}=0, \quad K_{3}=0, \quad K_{4}=-J_{3} / I_{2}=-\tau, \quad K_{5}=-I_{2}=-\kappa, \quad K_{6}=0 .
$$

Substituting these expressions into (3.18) and letting $F$ range over the other jet coordinates produces the non-phantom recurrence formulae

$$
\begin{array}{lll}
I_{3}=\mathcal{D} I_{2}, & \\
I_{4}=\mathcal{D} I_{3}+3 I_{2}^{3}-J_{3}^{2} / I_{2}, & J_{4}=\mathcal{D} J_{3}+I_{3} J_{3} / I_{2}, \\
I_{5}=\mathcal{D} I_{4}+10 I_{2}^{2} I_{3}-J_{3} J_{4} / I_{2}, & J_{5}=\mathcal{D} J_{4}+6 I_{2}^{2} J_{3}-J_{3} I_{4} / I_{2},
\end{array}
$$

and so on. Starting with (3.16), and successively substituting into (3.19), we find

$$
\begin{array}{lll}
I_{2}=\kappa, & \\
I_{3}=\kappa_{s}, & J_{3}=\kappa \tau, \\
I_{4}=\kappa_{s s}+3 \kappa^{3}-\kappa \tau^{2}, & J_{4}=\kappa \tau_{s}+2 \kappa_{s} \tau, \\
I_{5}=\kappa_{s s s s}-3 \kappa \tau \tau_{s}-3 \kappa_{s} \tau^{2}+19 \kappa^{2} \kappa_{s}, & J_{5}=\kappa \tau_{s s}+3 \kappa_{s} \tau_{s}+3 \kappa_{s s} \tau-\kappa \tau^{3}+9 \kappa^{3} \tau .
\end{array}
$$

This implies that the Euclidean normal form of a space curve has Taylor expansion

$$
\begin{aligned}
u_{0}(x)=\frac{1}{2} \kappa x^{2}+\frac{1}{6} \kappa_{s} x^{3} & +\frac{1}{24}\left(\kappa_{s s}+3 \kappa^{3}-\kappa \tau^{2}\right) x^{4}+ \\
& +\frac{1}{120}\left(\kappa_{\text {ssss }}-3 \kappa \tau \tau_{s}-3 \kappa_{s} \tau^{2}+19 \kappa^{2} \kappa_{s}\right) x^{5}+\cdots, \\
v_{0}(x)=\frac{1}{6} \kappa \tau x^{3}+\frac{1}{24}\left(2 \tau \kappa_{s}\right. & \left.+\kappa \tau_{s}\right) x^{4}+ \\
& +\frac{1}{120}\left(\kappa \tau_{s s}+3 \kappa_{s} \tau_{s}+3 \kappa_{s s} \tau-\kappa \tau^{3}+9 \kappa^{3} \tau\right) x^{5}+\cdots .
\end{aligned}
$$

Observe that if $\tau \equiv 0$, so that the curve is planar, then the first equation in (3.21) reduces to the planar normal form (2.33).

Example 3.2. Finally, we treat the action of the Euclidean group SE(3) on twodimensional surfaces $S \subset M=\mathbb{R}^{3}$. Now $p=2$, and we use coordinates $z=(x, y, u)$ on $M$. As usual, we focus our attention to surfaces given by the graphs of functions: $u=u(x, y)$. All our results can be readily adapted to general parametrized surfaces, and, as always, the final recurrence formulae make no reference to the underlying parametrization. We refer to $[\mathbf{1 4}, \mathbf{2 1}]$ for additional details. The surface jet space has coordinates

$$
\left(x, y, u, u_{x}, u_{y}, u_{x x}, u_{x y}, u_{y y}, u_{x x x}, u_{x x y}, u_{x y y}, u_{y y y}, \ldots\right) \text {, }
$$

and, in general, we use $u_{j k}$ to denote the jet coordinate corresponding to the partial derivative $\partial^{j+k} u / \partial x^{j} \partial y^{k}$. 
The classical moving frame construction, $[\mathbf{9}]$, relies on the coordinate cross-section

$$
x=y=u=u_{x}=u_{y}=u_{x y}=0 .
$$

The corresponding phantom invariants are

$$
\begin{aligned}
& \iota(x)=0, \quad \iota(y)=0, \quad I_{00}=\iota(u)=0, \\
& I_{10}=\iota\left(u_{x}\right)=0, \quad I_{01}=\iota\left(u_{y}\right)=0, \quad I_{11}=\iota\left(u_{x y}\right)=0,
\end{aligned}
$$

where, in general, we denote the normalized differential invariants by

$$
I_{j k}=\iota\left(u_{j k}\right), \quad j, k \geq 0 .
$$

The fundamental differential invariants of lowest order are the principal curvatures

$$
\kappa_{1}=I_{20}=\iota\left(u_{x x}\right), \quad \kappa_{2}=I_{02}=\iota\left(u_{y y}\right),
$$

and it can be shown - through inspection of the recurrence formulae - that they generate the algebra of differential invariants via invariant differentiation. Surprisingly, as explained below, they do not form a minimal generating set.

The selected cross-section (3.22) corresponds to translating and rotating the surface so that it acquires the Euclidean normal form by passing through the origin, having horizontal tangent plane, and so that the directions of principal curvature line up with the coordinate axes. This requires that the point $z \in S$ be non-umbilic, meaning that the two principal curvatures are unequal, $\kappa_{1} \neq \kappa_{2}$, which is the standard non-degeneracy condition required for the existence of a well-defined Euclidean moving frame, [9]. (At a non-degenerate umbilic, one could, in principle, employ a higher order moving frame.) The mean and Gaussian curvature invariants

$$
H=\frac{1}{2}\left(\kappa_{1}+\kappa_{2}\right), \quad K=\kappa_{1} \kappa_{2},
$$

are often used as convenient alternatives to the principal curvature invariants, since they eliminate some of the residual discrete ambiguities in the moving frame. The resulting left moving frame consists of the point on the curve defining the translation component $a=z \in \mathbb{R}^{3}$, while the columns of the rotation matrix $R=\left[\mathbf{t}_{1}, \mathbf{t}_{2}, \mathbf{n}\right] \in \mathrm{SO}(3)$ contain the unit tangent vectors $\mathbf{t}_{1}, \mathbf{t}_{2}$ forming the Darboux frame on the surface, $[\mathbf{9}]$, along with the unit normal $\mathbf{n}$.

Higher order differential invariants are obtained by differentiation with respect to the diagonalizing dual Darboux coframe $\varpi^{1}=\iota(d x), \varpi^{2}=\iota(d y)$. We let $\mathcal{D}_{1}, \mathcal{D}_{2}$ denote the dual invariant differential operators, which differentiate in the principal curvature directions, and defined so that the differential of any differential function $F$ can be written in invariant form

$$
d F=\left(\mathcal{D}_{1} F\right) \varpi^{1}+\left(\mathcal{D}_{2} F\right) \varpi^{2} .
$$

The invariant differential operators do not commute, but, rather satisfy the commutation relation

$$
\left[\mathcal{D}_{1}, \mathcal{D}_{2}\right]=\mathcal{D}_{1} \mathcal{D}_{2}-\mathcal{D}_{2} \mathcal{D}_{1}=Y_{2} \mathcal{D}_{1}-Y_{1} \mathcal{D}_{2}
$$


where

$$
Y_{1}=\frac{\kappa_{2,1}}{\kappa_{1}-\kappa_{2}}, \quad Y_{2}=\frac{\kappa_{1,2}}{\kappa_{2}-\kappa_{1}}
$$

are known as the commutator invariants, whose expressions can also be established using the full moving frame calculus, [14]. Note that the denominator in (3.28) vanishes at umbilic points on the surface, where the principal curvatures coincide $\kappa_{1}=\kappa_{2}$, and the moving frame is not valid.

Setting $F$ to be, successively, $x, y, u_{j k}$ in the general formulae (3.9) produces the recurrence relations

$$
\begin{array}{llll}
1=-\sum_{\sigma=1}^{6} K_{1}^{\sigma} \iota\left(\xi_{\sigma}\right), & 0=-\sum_{\sigma=1}^{6} K_{1}^{\sigma} \iota\left(\eta_{\sigma}\right), & I_{j+1, k}=\mathcal{D}_{1} I_{j k}-\sum_{\sigma=1}^{6} K_{1}^{\sigma} \iota\left(\varphi_{\sigma}^{j k}\right), & \\
0=-\sum_{\sigma=1}^{6} K_{2}^{\sigma} \iota\left(\xi_{\sigma}\right), & 1=-\sum_{\sigma=1}^{6} K_{2}^{\sigma} \iota\left(\eta_{\sigma}\right), & I_{j, k+1}=\mathcal{D}_{2} I_{j k}-\sum_{\sigma=1}^{6} K_{2}^{\sigma} \iota\left(\varphi_{\sigma}^{j k}\right), & j, k \geq 0,
\end{array}
$$

where $K_{1}^{\sigma}, K_{2}^{\sigma}$ are the Maurer-Cartan invariants, while $\xi_{\sigma}, \eta_{\sigma}, \varphi_{\sigma}^{j k}$ are, respectively, the coefficients of $\partial_{x}, \partial_{y}, \partial_{u_{j k}}$ in the prolonged infinitesimal generators, which are calculated via (3.8):

$$
\begin{aligned}
& \operatorname{pr} \mathbf{v}_{1}=\partial_{x}, \quad \operatorname{pr} \mathbf{v}_{2}=\partial_{y}, \quad \operatorname{pr} \mathbf{v}_{3}=\partial_{u}, \\
& \begin{aligned}
\operatorname{pr} \mathbf{v}_{4}=-y \partial_{x} & +x \partial_{y}-u_{y} \partial_{u_{x}}+u_{x} \partial_{u_{y}} \\
& -2 u_{x y} \partial_{u_{x x}}+\left(u_{x x}-u_{y y}\right) \partial_{u_{x y}}-2 u_{x y} \partial_{u_{y y}}+\cdots,
\end{aligned} \\
& \begin{aligned}
\operatorname{pr} \mathbf{v}_{5}=-u \partial_{x} & +x \partial_{u}+\left(1+u_{x}^{2}\right) \partial_{u_{x}}+u_{x} u_{y} \partial_{u_{y}} \\
& +3 u_{x} u_{x x} \partial_{u_{x x}}+\left(u_{y} u_{x x}+2 u_{x} u_{x y}\right) \partial_{u_{x y}}+\left(2 u_{y} u_{x y}+u_{x} u_{y y}\right) \partial_{u_{y y}}+\cdots,
\end{aligned} \\
& \operatorname{pr} \mathbf{v}_{6}=-u \partial_{y}+y \partial_{u}+u_{x} u_{y} \partial_{u_{x}}+\left(1+u_{y}^{2}\right) \partial_{u_{y}} \\
& +\left(u_{y} u_{x x}+2 u_{x} u_{x y}\right) \partial_{u_{x x}}+\left(2 u_{y} u_{x y}+u_{x} u_{y y}\right) \partial_{u_{x y}}+3 u_{y} u_{y y} \partial_{u_{y y}}+\cdots
\end{aligned}
$$

Substituting (3.30) into the recurrence formulae (3.29) corresponding to the phantom invariants (3.15), and solving the resulting linear systems produces the formulae for the Maurer-Cartan invariants

$$
\begin{aligned}
& K_{1}^{1}=-1, \quad K_{1}^{2}=0, \quad K_{1}^{3}=0, \quad K_{1}^{4}=-Y_{1}, \quad K_{1}^{5}=-\kappa_{1}, \quad K_{1}^{6}=0, \\
& K_{2}^{1}=0, \quad K_{2}^{2}=-1, \quad K_{2}^{3}=0, \quad K_{2}^{4}=-Y_{2}, \quad K_{2}^{5}=0, \quad K_{2}^{6}=-\kappa_{2} \text {. }
\end{aligned}
$$

Substituting these expressions back into (3.29), we successively obtain the desired formulae for the higher order normalized differential invariants in terms of the principal curvatures, of which the third order ones are

$$
I_{30}=\mathcal{D}_{1} \kappa_{1}=\kappa_{1,1}, \quad I_{21}=\mathcal{D}_{2} \kappa_{1}=\kappa_{1,2}, \quad I_{12}=\mathcal{D}_{1} \kappa_{2}=\kappa_{2,1}, \quad I_{03}=\mathcal{D}_{2} \kappa_{2}=\kappa_{2,2}
$$


while, taking these into account, the fourth order recurrence relations yield

$$
\begin{aligned}
& I_{40}=\kappa_{1,11}-\frac{3 \kappa_{1,2}^{2}}{\kappa_{1}-\kappa_{2}}+3 \kappa_{1}^{3}, \\
& I_{31}=\kappa_{1,12}-\frac{3 \kappa_{1,2} \kappa_{2,1}}{\kappa_{1}-\kappa_{2}}=\kappa_{1,21}+\frac{\kappa_{1,1} \kappa_{1,2}-2 \kappa_{1,2} \kappa_{2,1}}{\kappa_{1}-\kappa_{2}}, \\
& I_{22}=\kappa_{1,22}+\frac{\kappa_{1,1} \kappa_{2,1}-2 \kappa_{2,1}^{2}}{\kappa_{1}-\kappa_{2}}+\kappa_{1} \kappa_{2}^{2}=\kappa_{2,11}-\frac{\kappa_{1,2} \kappa_{2,2}-2 \kappa_{1,2}^{2}}{\kappa_{1}-\kappa_{2}}+\kappa_{1}^{2} \kappa_{2}, \\
& I_{13}=\kappa_{2,21}+\frac{3 \kappa_{1,2} \kappa_{2,1}}{\kappa_{1}-\kappa_{2}}=\kappa_{2,12}-\frac{\kappa_{2,1} \kappa_{2,2}-2 \kappa_{1,2} \kappa_{2,1}}{\kappa_{1}-\kappa_{2}}, \\
& I_{04}=\kappa_{2,22}+\frac{3 \kappa_{2,1}^{2}}{\kappa_{1}-\kappa_{2}}+3 \kappa_{2}^{3} .
\end{aligned}
$$

There are two distinct formulae for $I_{31}, I_{22}, I_{13}$ because they appear in both the first and second set of recurrence formulae in (3.29). The two expressions for $I_{31}$ and $I_{13}$ agree owing to the non-commutativity, $(3.27)$, of $\mathcal{D}_{1}, \mathcal{D}_{2}$, while the two expressions for $I_{22}$ imply the celebrated Codazzi syzygy

$$
\kappa_{1,22}-\kappa_{2,11}+\frac{\kappa_{1,1} \kappa_{2,1}+\kappa_{1,2} \kappa_{2,2}-2 \kappa_{2,1}^{2}-2 \kappa_{1,2}^{2}}{\kappa_{1}-\kappa_{2}}-\kappa_{1} \kappa_{2}\left(\kappa_{1}-\kappa_{2}\right)=0,
$$

which can be written compactly as

$$
K=\kappa_{1} \kappa_{2}=-\left(\mathcal{D}_{1}+Y_{1}\right) Y_{1}-\left(\mathcal{D}_{2}+Y_{2}\right) Y_{2} .
$$

The latter is the key identity employed by Guggenheimer, [9], for a short proof of Gauss' Theorema Egregium. We conclude that the Euclidean normal form of a surface $z=u(x, y)$ at a non-umbilic point is

$$
\begin{array}{r}
u(x, y)=\frac{1}{2} \kappa_{1} x^{2}+\frac{1}{2} \kappa_{2} y^{2}+\frac{1}{6} \kappa_{1,1} x^{3}+\frac{1}{2} \kappa_{1,2} x^{2} y+\frac{1}{2} \kappa_{2,1} x y^{2}+\frac{1}{6} \kappa_{2,2} y^{3}+ \\
+\frac{1}{24} I_{40} x^{4}+\frac{1}{6} I_{31} x^{3} y+\frac{1}{4} I_{22} x^{2} y^{2}+\frac{1}{6} I_{13} x y^{3}+\frac{1}{24} I_{04} y^{4}+\cdots,
\end{array}
$$

where the fourth order coefficients appear in (3.33). Higher order terms can easily and automatically be determined using the recurrence formulae.

It is a classical result that the algebra of Euclidean differential invariants of a nonumbilic surface $S \subset \mathbb{R}^{3}$ is generated, through invariant differentiation, by the principal curvatures or, equivalently, the Gauss and mean curvatures; see [9] and, for a direct proof based on the moving frame recurrence relations, [14]. Surprisingly, as noted in [21], for suitably nondegenerate surfaces, the mean curvature by itself suffices to generate all the differential invariants. In particular, the Gauss curvature $K$ can be written as an explicit universal rational combination of the invariant derivatives of the mean curvature $H$ of order $\leq 4$. Here we go slightly further by completely characterizing the nondegeneracy condition found in $[\mathbf{2 1}]$.

Definition 3.3. A surface $S \subset \mathbb{R}^{3}$ is mean curvature degenerate if, for any nonumbilic point $z_{0} \in S$, there exist scalar functions $f_{1}(t), f_{2}(t)$, such that

$$
\mathcal{D}_{1} H=f_{1}(H), \quad \mathcal{D}_{2} H=f_{2}(H),
$$


at all points $z \in S$ in a suitable neighborhood of $z_{0}$.

Clearly any constant mean curvature surface is mean curvature degenerate, with $f_{1}(t) \equiv f_{2}(t) \equiv 0$. Surfaces with non-constant mean curvature that admit a one-parameter group of Euclidean symmetries, i.e., non-cylindrical or non-spherical surfaces of rotation, non-planar surfaces of translation, or helicoid surfaces, obtained by, respectively, rotating, translating, or screwing a plane curve, are also mean curvature degenerate since, by the signature characterization of symmetry groups, $[4]$, they have exactly one non-constant functionally independent differential invariant, namely their mean curvature $H$ and hence any other differential invariant, including the invariant derivatives of $H$ - as well as the Gauss curvature $K$ - must be functionally dependent upon $H$. There also exist surfaces without continuous symmetries that are, nevertheless, mean curvature degenerate since it is entirely possible that (3.37) holds, but the Gauss curvature remains functionally independent of $H$. However, I do not know whether there is a good intrinsic geometric characterization of such surfaces.

Theorem 3.4. If a surface is mean curvature nondegenerate then the algebra of differential invariants is generated entirely by the mean curvature and its successive invariant derivatives.

Proof: Following the arguments in [21], in view of the Codazzi formula (3.35), it suffices to write the commutator invariants $Y_{1}, Y_{2}$ in terms of the mean curvature. To this end, we note that the commutator identity (3.27) can be applied to any differential invariant. In particular,

$$
\mathcal{D}_{1} \mathcal{D}_{2} H-\mathcal{D}_{2} \mathcal{D}_{1} H=Y_{2} \mathcal{D}_{1} H-Y_{1} \mathcal{D}_{2} H
$$

and, furthermore, for $j=1$ or 2 ,

$$
\mathcal{D}_{1} \mathcal{D}_{2} \mathcal{D}_{j} H-\mathcal{D}_{2} \mathcal{D}_{1} \mathcal{D}_{j} H=Y_{2} \mathcal{D}_{1} \mathcal{D}_{j} H-Y_{1} \mathcal{D}_{2} \mathcal{D}_{j} H
$$

Provided the nondegeneracy condition

$$
\left(\mathcal{D}_{1} H\right)\left(\mathcal{D}_{2} \mathcal{D}_{j} H\right) \neq\left(\mathcal{D}_{2} H\right)\left(\mathcal{D}_{1} \mathcal{D}_{j} H\right), \quad \text { for } j=1 \text { or } 2,
$$

holds, we can solve (3.38-39) to write the commutator invariants $Y_{1}, Y_{2}$ as explicit rational functions of invariant derivatives of $H$. Plugging these expressions into the right hand side of the Codazzi identity (3.35) produces an explicit formula for the Gauss curvature as a rational function of the invariant derivatives, of order $\leq 4$, of the mean curvature, which is valid for all surfaces satisfying the nondegeneracy condition (3.40).

Thus it remains to show that (3.40) is equivalent to mean curvature nondegeneracy of the surface. First, if (3.37) holds, then

$$
\mathcal{D}_{i} \mathcal{D}_{j} H=\mathcal{D}_{i} f_{j}(H)=f_{j}^{\prime}(H) \mathcal{D}_{i} H=f_{j}^{\prime}(H) f_{i}(H), \quad i, j=1,2 .
$$

This immediately implies

$$
\left(\mathcal{D}_{1} H\right)\left(\mathcal{D}_{2} \mathcal{D}_{j} H\right)=\left(\mathcal{D}_{2} H\right)\left(\mathcal{D}_{1} \mathcal{D}_{j} H\right), \quad j=1,2,
$$


proving mean curvature degeneracy. Vice versa, in view of formula (3.26), the degeneracy condition (3.41) implies that, for each $j=1,2$, the differentials $d H, d\left(\mathcal{D}_{j} H\right)$ are linearly dependent everywhere on $S$, which, by a general theorem characterizing functional dependency, [17; Theorem 2.16], implies that, locally, $\mathcal{D}_{j} H$ can be written as a function of $H$, thus establishing the degeneracy condition (3.37).

Q.E.D.

\section{References}

[1] Bruce, J.W., and Giblin, P.J., Curves and Singularities, Cambridge University Press, Cambridge, 1984.

[2] Calabi, E., Olver, P.J., Shakiban, C., Tannenbaum, A., and Haker, S., Differential and numerically invariant signature curves applied to object recognition, Int. J. Computer Vision 26 (1998), 107-135.

[3] Cipolla, R., and Giblin, P., Visual Motion of Curves and Surfaces, Cambridge University Press, Cambridge, 2000.

[4] Fels, M., and Olver, P.J., Moving coframes. II. Regularization and theoretical foundations, Acta Appl. Math. 55 (1999), 127-208.

[5] Gray, A., Abbena, E., and Salamon, S., Modern Differential Geometry of Curves and Surfaces with Mathematica, 3rd ed., Chapman \& Hall/CRC, Boca Raton, Fl., 2006.

[6] Green, M.L., The moving frame, differential invariants and rigidity theorems for curves in homogeneous spaces, Duke Math. J. 45 (1978), 735-779.

[7] Grim, A., O'Connor, T., Olver, P.J., Shakiban, C., Slechta, R., and Thompson, R., Automatic reassembly of three-dimensional jigsaw puzzles, Int. J. Image Graphics 16 (2016), 1650009.

[8] Grim, A., and Shakiban, C., Applications of signatures in diagnosing breast cancer, Minnesota J. Undergrad. Math. 1 (1) (2015), 001.

[9] Guggenheimer, H.W., Differential Geometry, McGraw-Hill, New York, 1963.

[10] Hoff, D., and Olver, P.J., Automatic solution of jigsaw puzzles, J. Math. Imaging Vision 49 (2014), 234-250.

[11] Hubert, E., and Kogan, I.A., Rational invariants of a group action. Construction and rewriting, J. Symb. Comp. 42 (2007), 203-217.

[12] Hubert, E., and Olver, P.J., Differential invariants of conformal and projective surfaces, SIGMA 3 (2007), 097.

[13] Kim, P., Invariantization of numerical schemes using moving frames, BIT 47 (2007), $525-546$.

[14] Kogan, I.A., and Olver, P.J., Invariant Euler-Lagrange equations and the invariant variational bicomplex, Acta Appl. Math. 76 (2003), 137-193.

[15] Mansfield, E.L., Algorithms for symmetric differential systems, Found. Comput. Math. 1 (2001), 335-383. 
[16] Marsland, S., and McLachlan, R.I., Möbius invariants of shapes and images, SIGMA: Symmetry Integrability Geom. Methods Appl. 12 (2016), 080.

[17] Olver, P.J., Applications of Lie Groups to Differential Equations, Second Edition, Graduate Texts in Mathematics, vol. 107, Springer-Verlag, New York, 1993.

[18] Olver, P.J., Equivalence, Invariants, and Symmetry, Cambridge University Press, 1995.

[19] Olver, P.J., Moving frames and singularities of prolonged group actions, Selecta Math. 6 (2000), 41-77.

[20] Olver, P.J., Joint invariant signatures, Found. Comput. Math. 1 (2001), 3-67.

[21] Olver, P.J., Differential invariants of surfaces, Diff. Geom. Appl. 27 (2009), 230-239.

[22] Olver, P.J., Lectures on moving frames, in: Symmetries and Integrability of Difference Equations, D. Levi, P. Olver, Z. Thomova, and P. Winternitz, eds., London Math. Soc. Lecture Note Series, vol. 381, Cambridge University Press, Cambridge, 2011, pp. 207-246.

[23] Olver, P.J., and Pohjanpelto, J., Moving frames for Lie pseudo-groups, Canadian J. Math. 60 (2008), 1336-1386.

[24] Olver, P.J., and Pohjanpelto, J., Differential invariant algebras of Lie pseudo-groups, Adv. Math. 222 (2009), 1746-1792.

[25] Shakiban, C., and Lloyd, P., Signature curves statistics of DNA supercoils, in: Geometry, Integrability and Quantization, vol. 5, I.M. Mladenov and A.C. Hirschfeld, eds., Softex, Sofia, Bulgaria, 2004, pp. 203-210. 